\title{
Use of fast-sequence spine MRI in pediatric patients
}

\author{
Jordan I. Gewirtz, BS, ${ }^{1}$ Alex Skidmore, BS, ${ }^{1}$ Matthew D. Smyth, MD, ${ }^{1}$ \\ David D. Limbrick Jr., MD, PhD, ${ }^{1}$ Manu Goyal, MD, MSc, ${ }^{2}$ Joshua S. Shimony, MD, PhD, ${ }^{2}$ \\ Robert C. McKinstry, MD, PhD, ${ }^{2}$ Mari L. Groves, MD, ${ }^{3}$ and Jennifer M. Strahle, MD ${ }^{1}$
}

1Department of Neurological Surgery and ${ }^{2}$ Mallinckrodt Institute of Radiology, Washington University School of Medicine, St. Louis, Missouri; and ${ }^{3}$ Department of Neurosurgery, Johns Hopkins School of Medicine, Baltimore, Maryland

\begin{abstract}
OBJECTIVE The immediate and long-term risk of anesthesia in the pediatric population is controversial. Traditional spine MRI protocols require the patient to remain still during the examination, and in young children this frequently results in the need for sedation administration. The authors' goal was to develop an abbreviated spine MRI protocol to reduce sedation administration in young patients undergoing spine MRI.
\end{abstract}

METHODS After IRB approval, the medical records of all pediatric patients who underwent a fast spine MRI protocol between 2017 and 2019 were reviewed. The protocol consisted of T2-weighted half-Fourier acquisition single-shot turbo spin echo, T1-weighted turbo spin echo, and T2-weighted STIR sequences acquired in the sagittal plane. The total acquisition time was 2 minutes with no single sequence acquisition longer than 60 seconds. Interpretability of the scans was assessed in accordance with the radiology report in conjunction with the neurosurgeon's clinical notes.

RESULTS A total of 47 fast spine MRI sessions were performed in 45 patients. The median age at the time of the MRI was 2.4 years (25th-75th quartile, 1.1-4.3 years; range $0.16-18.58$ years). The most common indication for imaging was to rule out or follow a known syrinx $(n=30)$, followed by the need to rule out or follow known spinal dysraphism $(n=22)$. There were no uninterpretable or unusable scans. Eight of 47 scans were noted to have moderate motion artifact limitations with respect to the quality of the scan. Seven patients underwent a subsequent MRI with a sedated standard spine protocol within 1 year from the fast scan, which confirmed the findings on the fast MRI protocol with no new findings identified.

CONCLUSIONS The authors report the first pediatric series of a fast spine MRI protocol for use in young patients. The protocol does not require sedation and is able to identify and monitor syrinx, spinal dysraphism, and potentially other intraspinal anomalies.

https://thejns.org/doi/abs/10.3171/2020.5.PEDS20137

KEYWORDS MRI; fast sequence; spine; sedation

$\mathrm{T}$ Raditional spine MRI protocols require the patient to remain still during the examination, frequently resulting in the need for sedation in young children. The immediate and long-term risk of anesthesia in the pediatric population is controversial. Studies suggesting the possibility of negative neurocognitive outcomes with prenatal or pediatric exposure to general anesthesia have led the US FDA to issue updated warnings and require label changes on general anesthesia and sedation medications regarding the dangers of repeated use in pregnant women and children younger than 3 years of age..$^{1-4}$ These warnings have led to increased interest in pediatric imaging modalities that do not require the use of sedation.
Fast-sequence MRI (fsMRI), with the reduction in the duration of imaging time, obviates the need for sedation and has been shown to be effective in pediatric patients in the surveillance and diagnosis of hydrocephalus and ventricular shunt malfunction. ${ }^{5-7}$ Similarly, an abbreviated MRI protocol has been successfully used in spinal imaging in adults to reduce the required acquisition time without sacrificing screening and diagnostic accuracy in lumbar spondylosis. ${ }^{8,9}$ We therefore investigated whether these benefits in speed could translate to spine imaging in pediatric patients without a prohibitive loss of diagnostic accuracy. We developed an abbreviated spine MRI protocol to evaluate the ability for fsMRI to provide adequate radiological resolution in spinal pathology diagnoses without sedation.

ABBREVIATIONS fSMRI = fast-sequence MRI; HASTE = half-Fourier acquisition single-shot turbo spin echo; IPAT = integrated parallel imaging technique; SPACE = sampling perfection with application-optimized contrasts using different flip-angle evolutions.

SUBMITTED February 24, 2020. ACCEPTED May 18, 2020.

INCLUDE WHEN CITING Published online September 18, 2020; DOI: 10.3171/2020.5.PEDS20137. 


\section{Methods}

Following institutional review board approval at the Washington University School of Medicine, demographic, clinical, and radiological records of all pediatric patients who underwent a spine fsMRI protocol between 2017 and 2019 were reviewed. The protocol consisted of T2weighted half-Fourier acquisition single-shot turbo spin echo (HASTE; scan time 15 seconds, voxel size $0.7 \times 0.7$ $\times 4.0 \mathrm{~mm}$, FOV adjustable to cover full spine, TR 1000 $\mathrm{msec}$, TE $78 \mathrm{msec}$, integrated parallel imaging technique [IPAT] off, partial-phase Fourier 4/8); T1-weighted turbo spin echo (scan time 45 seconds, voxel size $1.1 \times 1.1 \times 4.0$ $\mathrm{mm}$, FOV adjustable to cover full spine, TR $545 \mathrm{msec}$, TE 9.4 msec, IPAT 2, partial phase off); and T2-weighted STIR (scan time 59 seconds, voxel size $1.1 \times 1.1 \times 4.0 \mathrm{~mm}$, FOV adjustable to cover full spine, TR $4080 \mathrm{msec}$, TE 64 msec, IPAT 3, partial phase off) imaging sessions. All sequences were acquired without sedation in the sagittal plane with an adjustable FOV to cover the full spine on a Siemens Aera 1.5-T MRI machine at St. Louis Children's Hospital. The total acquisition time was 2 minutes with no single sequence longer than 60 seconds. Prior to imaging, the potential limitations of fsMRI were discussed with the patient and family. The interpretability and quality of the scans were assessed in accordance with the radiology report in conjunction with the neurosurgeon's clinical notes. Patients who subsequently received a standard follow-up MRI protocol were identified and those images were compared to their prior images from the fsMRI. Neither the spine fsMRI nor the standard follow-up MRI protocol was reviewed in a blinded fashion because the interpretations of the images were taken from the medical records.

\section{Results}

Forty-seven spine fsMRI studies were performed in 45 patients (27 male, 18 female) over the 2 -year period. The median age at time of MRI was 2.4 years $(25$ th-75th quartile, $1.1-4.3$ years; range $0.16-18.58$ years). The median patient age at initial presentation to the clinic was 0.8 years (25th-75th quartile, $0.19-4.15$ years; range $0.03-18.55$ years). Twenty-one patients had a clinical diagnosis of hydrocephalus, and 26 patients had a previous neurosurgical procedure; the most common surgeries included myelomeningocele repair, shunt placements and revisions, Chiari decompression, and surgical treatment for craniosynostosis. Nineteen patients were previously imaged using a standard spine MRI protocol. The choice of sedative was left to the treating anesthesiologist. Table 1 depicts the indications for the 19 patients who underwent prior spinal MRI. Thirty-nine of 47 fsMRI studies included the entire spine (Table 2). The most common in-

TABLE 1. Indication for imaging assessed by prior standard MRI

\begin{tabular}{lc}
\hline \multicolumn{1}{c}{ Potential Pathology } & Count \\
\hline Syrinx & 8 \\
\hline Spinal dysraphism & 3 \\
\hline Syrinx \& spinal dysraphism & 8 \\
\hline
\end{tabular}

TABLE 2. Segment of spine imaged with fsMRI

\begin{tabular}{lc}
\hline \multicolumn{1}{c}{ Area Scanned } & Count \\
\hline Cervical spine & 2 \\
\hline Thoracic spine & 3 \\
\hline Lumbar spine & 2 \\
\hline Thoracic lumbar spine & 1 \\
\hline Total spine & 39 \\
\hline
\end{tabular}

dication for imaging was to rule out or follow a known syrinx $(n=30)$, followed by the need to rule out or follow known spinal dysraphism $(n=22)$ (Table 3). Table 4 presents the radiological findings identified by fsMRI in patients evaluated for spinal dysraphism. Seventeen of 47 fsMRI studies were conducted due to 19 symptomatic clinical concerns (Table 5); 19 were for routine monitoring with no symptomatic changes, and 11 were to rule out a potential pathology with no symptomatic changes. The fsMRI study alone was not used for surgery planning, nor did it directly lead to surgery, and it was not used independently postoperatively. Representative sagittal images of the fsMRI session are depicted in Fig. 1. Eight of 47 scans were noted to have moderate motion artifact limitations with respect to the quality of the scan, but there were no uninterpretable or unusable scans (Fig. 2). Seven of the 47 rapid scans were followed with a subsequent sedated standard spine MRI protocol within 1 year of the rapid scan: 4 for a more detailed evaluation of syrinx, 2 for routine monitoring, and 1 for an unrelated surgical follow-up (Fig. 3). Two of 7 standard protocol follow-up MRI studies were conducted due to clinical concerns-developmental delays and guarded movements. The 5 remaining studies were for more detailed evaluations in conjunction with routine monitoring. All 7 subsequent sedated MRI studies confirmed the findings on the fsMRI with no new findings identified.

\section{Discussion}

The aim of this study was to report in a retrospective, observational fashion on the early use of a nonsedated fsMRI protocol in the assessment of pediatric spinal pathology. In all but 4 of the 47 instances of replacing standard MRI with fsMRI, the radiological report indicated that the images acquired were sufficient for the indicated diagnostic purpose. This included fsMRI studies showing no abnormalities, in which the scan was deemed suitable to assess for intraspinal pathology. In those 4 remaining cases, the initial scan was deemed informative, but the

TABLE 3. Indication for imaging performed by fsMRI

\begin{tabular}{lc}
\hline Potential Pathology & Count \\
\hline Syrinx & 24 \\
\hline Spinal dysraphism & 16 \\
\hline Syrinx \& spinal dysraphism & 6 \\
\hline Other & 1 \\
\hline
\end{tabular}


TABLE 4. Radiological findings identified by fsMRI in those evaluated for spinal dysraphism

\begin{tabular}{lc}
\hline Radiological Finding & Count \\
\hline Myelomeningocele & 8 \\
\hline Myelocystocele & 1 \\
\hline Lipomyelomeningocele & 1 \\
\hline Meningocele & 2 \\
\hline Diastematomyelia & 1 \\
\hline Hemivertebra & 1 \\
\hline Rudimentary disc & 1 \\
\hline Arachnoid cyst & 1 \\
\hline Spinal canal narrowing & 1 \\
\hline Kyphosis & 2 \\
\hline Lordosis & 1 \\
\hline Hemangioma & 1 \\
\hline No pathology identified & 3 \\
\hline
\end{tabular}

On 2 MRI studies, 2 pathologies were identified.

physician ordered a subsequent sedated scan for better resolution to confirm findings. There were no additional abnormalities identified on the standard MRI compared with the initial fsMRI. No patient required repeat spine fsMRI. Our initial findings with fsMRI are encouraging for the continued clinical use of the imaging protocol.

\section{Evolution of Shunt Protocol Imaging Modalities}

Whereas fsMRI for the spine in pediatric patients has not been reported, the use of this protocol for the brain has been well studied and is standard across many centers. Historically, CT scans were a frequently used imaging method for the evaluation of ventricle size. The motivation for the development of a shunt MRI protocol was to mitigate the related morbidities due to increased cumulative ionizing radiation exposure associated with repeated CT scans in a vulnerable pediatric population. ${ }^{10,11}$ Traditional brain MRI protocols avoid radiation but have long acquisition times and frequently require sedation in children. Thus, the need for rapid cranial shunt protocol MRI was critical in order for MRI to replace conventional CT imaging in this clinical setting.

The development of a cranial fsMRI method has been shown to be successful in routine surveillance and monitoring of pediatric hydrocephalus, including ventricular shunt malfunction, and has been adopted by most institu-

TABLE 5. Clinical concerns for fsMRI

\begin{tabular}{lc}
\hline \multicolumn{1}{c}{ Clinical Concern } & Count \\
\hline $\begin{array}{l}\text { Asymmetric gluteal cleft or cutaneous marker of spinal } \\
\text { dysraphism }\end{array}$ & 4 \\
\hline Changes in lower-extremity function, gait, or reflexes & 12 \\
\hline Headache or emesis & 3 \\
\hline
\end{tabular}

Two MRI studies were conducted for 2 clinical concerns.
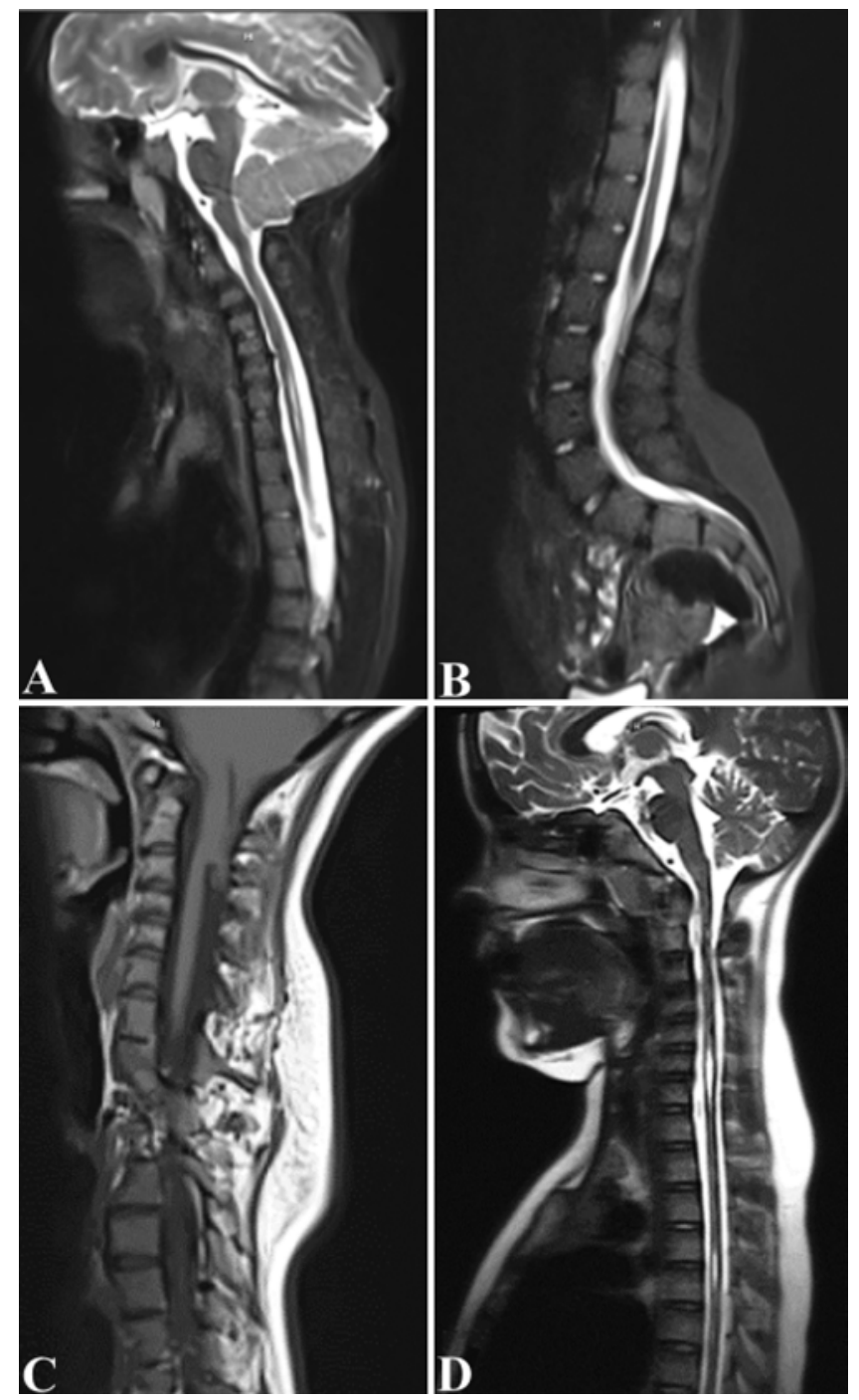

FIG. 1. Representative images acquired in 4 different patients with the fsMRI spine protocol with no motion artifact. A: T2-weighted sagittal image depicting the cervical and thoracic spine with a very small syrinx. B: T2-weighted sagittal image of the lower thoracic and lumbar spine with no appreciable pathology. C: T1-weighted sagittal image depicting the cervical and thoracic spine with low tonsil position and vertebral segmentation anomalies. D: T2-weighted sagittal image depicting the cervical and thoracic spine with a syrinx.

tions. $^{5-7}$

\section{Prior Fast Spine MRI Literature}

The current literature with respect to rapid spine MRI studies is very limited and has only reported on nonpediatric populations. ${ }^{1}$ The first series by Robertson et al. reported the use of fsMRI for lumbar spondylosis and consisted of two single-effective-echo long TR fast spin echo acquisition sequences: one in the sagittal plane and the other in the axial plane. ${ }^{8}$ The total scan time was less than 2.5 minutes, with each sequence lasting 1.18 minutes and 1.04 minutes for the sagittal and axial planes, respectively, compared with the standard protocol, in which the acquisition time was 28 minutes. 

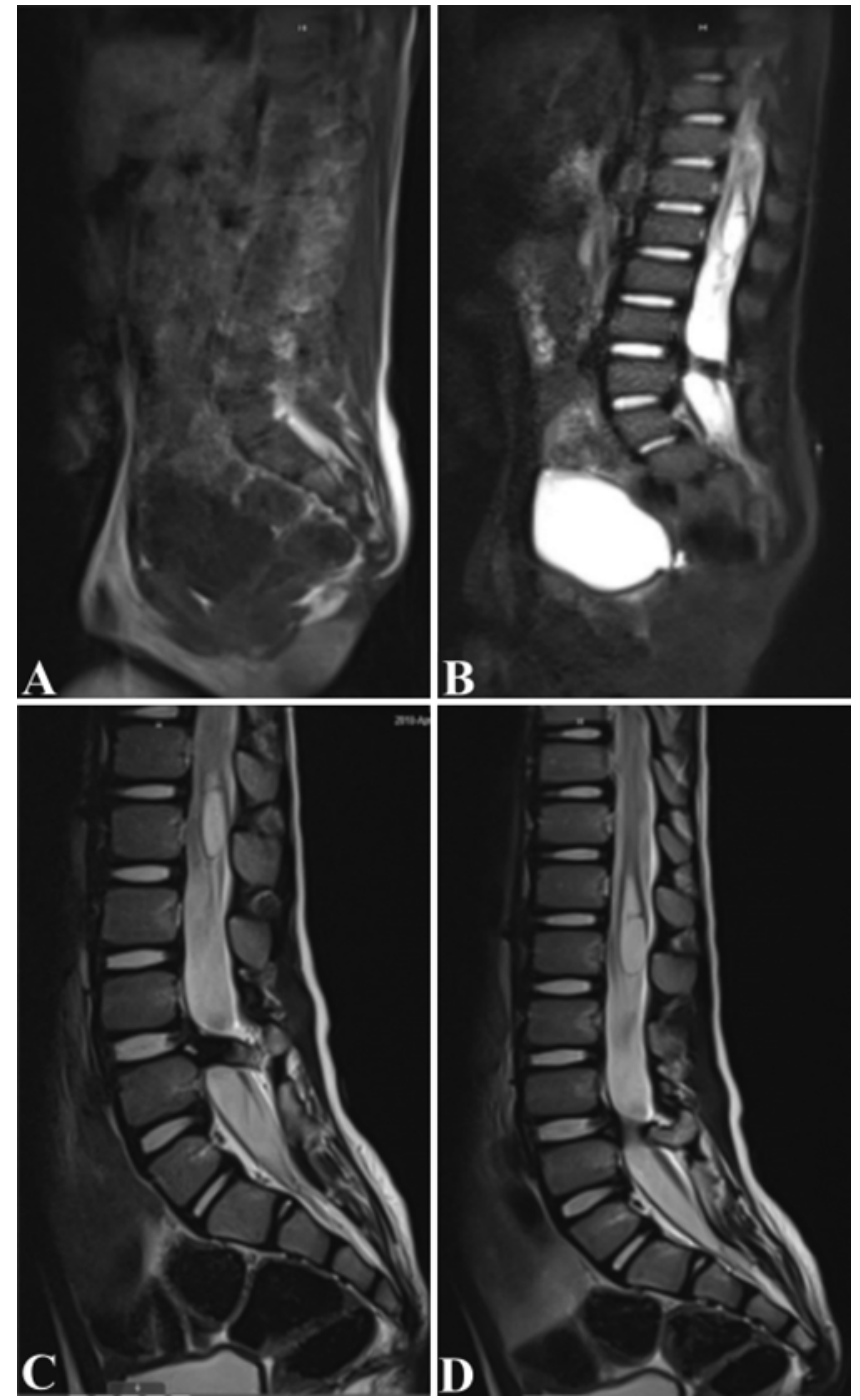

FIG. 2. Images acquired with the fsMRI spine protocol with motion artifact ( $A$ and $B$ ) and subsequent follow-up standard MRI ( $C$ and $D)$ of a 5 -year-old boy who has split cord malformation. A: T1-weighted sagittal image depicting the thoracic and lumbar spine with significant motion artifact. B: T2-weighted sagittal image depicting the thoracic and lumbar spine with mild motion artifact. C: T2-weighted sagittal image depicting the lumbar spine. D: T2-weighted sagittal image depicting the thoracic and lumbar spine.

More recently, computer-rendered 3D MR images have been used to shorten the image acquisition time. Sayah et al. report on the use of a rapid lumbar spine MRI using 3D T2-weighted SPACE imaging in the evaluation of degenerative changes of the lumbar spine. ${ }^{9}$ Sagittal T1- and T2-weighted SPACE sequences with axial SPACE reformations were used in their rapid protocol with a total scan time of 8 minutes, 46 seconds versus 18 minutes, 40 seconds for the standard protocol. The rapid and full studies had the following sensitivities and specificities respectively: $76.0 \%$ (rapid) and $69.3 \%$ (full) and $97.2 \%$ (rapid) and $97.9 \%$ (full) for all degenerative processes. For specific degenerative pathology, rapid and full sensitivities were as follows: disk herniation $68.7 \%$ and $66.3 \%$; canal compromise $85.2 \%$ and $81.5 \%$; lateral recess compromise $82.9 \%$

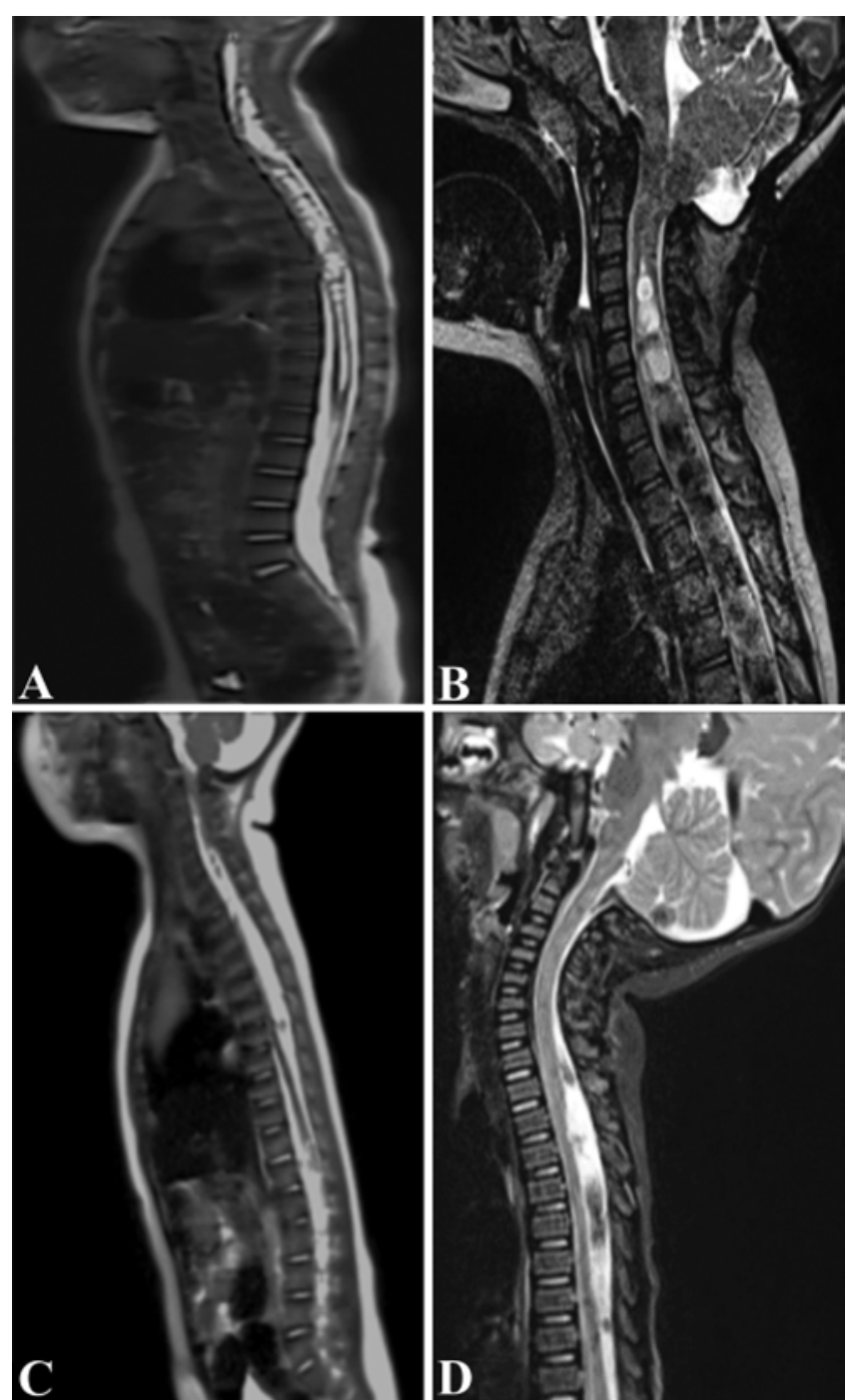

FIG. 3. T2-weighted images acquired with the fsMRI spine protocol (A and $C$ ) and subsequent follow-up standard MRI (B and D) in 2 sets of patients (case 1, A and B; case 2, C and D). A: Sagittal image depicting the total spine with a holocord syrinx. B: Sagittal image depicting the cervical and thoracic spine with a holocord syrinx. C: Sagittal image of the total spine showing a potential arachnoid cyst. D: Sagittal image of the cervical and thoracic spine showing a potential arachnoid cyst and anterior cord displacement.

and $69.1 \%$; and foraminal compromise $76.9 \%$ and $69.7 \%$. While isotropic SPACE T2-weighted imaging was able to image lumbar spondylosis, the authors found limitations for lesions in the spinal cord or soft tissue. Thus, further investigations are needed to determine for which conditions a rapid 3D MRI is suited.

\section{Fast Spine MRI}

Our fsMRI protocol results in a marked decrease in scan acquisition time compared with the standard MRI protocol (2 minutes compared with up to 60 minutes for images obtained with and without contrast for full spine), with minimal observed reduction in diagnostic utility for gross abnormalities such as syrinx and spinal dysraphism. 
For larger children approaching adulthood, the field of view would sometimes need to be split into 2 segments with 2 acquisition sequences to cover the full spine: the cervical and upper thoracic spine and the lumbar and lower thoracic spine. This modification to the procedure doubled the scan time; however, there was no reduction in resolution for these bigger patients. The fsMRI protocol reduces the time spent in the scanner and allows for more openings in the MRI schedule. The fsMRI billed is less than that for a standard MRI session. Syrinxes and spinal dysraphism are conditions ideally suited for this rapid protocol because, unlike many other pediatric conditions, the clinically significant abnormalities are larger than the minimum resolution of the scan. In this sense, fsMRI serves as a screening tool compared with the higher-resolution full-protocol MRI.

\section{Limitations and Future Directions}

Because this was not a paired study design, the ability to compare findings on fsMRI and sedated MRI is limited. However, we saw no new findings on any of the 7 subsequent MRI studies, and the diagnostic and monitoring utility of the sedated MRI was no greater than that of the fsMRI for the given indications. It should also be noted that a potential bias in the sample group is that clinically stable children may have been more likely to undergo the fsMRI. For some patients, parents may be present to provide coaching to minimize movement during the scan. In the context of the fsMRI, only syringomyelia and spinal dysraphism were assessed in our cohort. We are therefore unable to assess the ability of fsMRI of the spine to detect other pathologies. The T2 HASTE sequence had poor contrast sensitivity for cord pathologies but was excellent for CSF and cord differentiation. ${ }^{12}$ The protocol used here was performed on a Siemens Aera 1.5-T scanner and may or may not be replicable on other scanners. However, HASTE is a half-Fourier single-shot turbo spin echo acquisition sequence, and almost all MRI vendors have an equivalent sequence. We also acknowledge that new techniques for fast MRI, such as compressed sensing, are under development, which will likely further improve the speed and quality of fast MRI studies.

\section{Conclusions}

This report is the first series of fsMRI of the spine for use in pediatric patients. The protocol is able to successfully identify and monitor syrinx, spinal dysraphism, and potentially other intraspinal anomalies without sedation. Further validation and controlled, prospective studies are needed to assess fsMRI in pediatric patients with spinal pathology other than syrinx and spinal dysraphism.

\section{Acknowledgments}

Support was provided by the Eunice Kennedy Shriver National Institute of Child Health and Human Development of the NIH under award no. U54 HD087011 to the Intellectual and Developmental Disabilities Research Center at Washington (J.S.S.) and the Doris Duke Foundation (J.M.S.).

\section{References}

1. Rappaport BA, Suresh S, Hertz S, et al. Anesthetic neurotoxicity-clinical implications of animal models. $N$ Engl J Med. 2015;372(9):796-797.

2. US Food \& Drug Administration. FDA Drug Safety Communication: FDA approves label changes for use of general anesthetic and sedation drugs in young children. US Food \& Drug Administration; 2017. Accessed June 16, 2020. https: //www.fda.gov/drugs/drug-safety-and-availability/fda-drugsafety-communication-fda-approves-label-changes-usegeneral-anesthetic-and-sedation-drugs

3. US Food \& Drug Administration. FDA Drug Safety Communication: FDA review results in new warnings about using general anesthetics and sedation drugs in young children and pregnant women. US Food \& Drug Administration; 2016. Accessed June 16, 2020. https://www.fda.gov/drugs/ drug-safety-and-availability/fda-drug-safety-communicationfda-review-results-new-warnings-about-using-generalanesthetics-and

4. Yu CK, Yuen VM, Wong GT, Irwin MG. The effects of anaesthesia on the developing brain: a summary of the clinical evidence. F1000Res. 2013;2:166.

5. Ashley WW Jr, McKinstry RC, Leonard JR, et al. Use of rapid-sequence magnetic resonance imaging for evaluation of hydrocephalus in children. J Neurosurg. 2005;103(2)(suppl): 124-130.

6. Boyle TP, Paldino MJ, Kimia AA, et al. Comparison of rapid cranial MRI to CT for ventricular shunt malfunction. Pediatrics. 2014;134(1):e47-e54.

7. Patel DM, Tubbs RS, Pate G, et al. Fast-sequence MRI studies for surveillance imaging in pediatric hydrocephalus. $J$ Neurosurg Pediatr. 2014;13(4):440-447.

8. Robertson WD, Jarvik JG, Tsuruda JS, et al. The comparison of a rapid screening MR protocol with a conventional MR protocol for lumbar spondylosis. AJR Am J Roentgenol. 1996;166(4):909-916.

9. Sayah A, Jay AK, Toaff JS, et al. Effectiveness of a rapid lumbar spine MRI protocol using 3D T2-weighted SPACE imaging versus a standard protocol for evaluation of degenerative changes of the lumbar spine. AJR Am J Roentgenol. 2016;207(3):614-620.

10. Koral K, Blackburn T, Bailey AA, et al. Strengthening the argument for rapid brain MR imaging: estimation of reduction in lifetime attributable risk of developing fatal cancer in children with shunted hydrocephalus by instituting a rapid brain MR imaging protocol in lieu of head CT. AJNR Am J Neuroradiol. 2012;33(10):1851-1854.

11. Miglioretti DL, Johnson E, Williams A, et al. The use of computed tomography in pediatrics and the associated radiation exposure and estimated cancer risk. JAMA Pediatr. 2013;167(8):700-707.

12. Sugahara T, Korogi Y, Hirai T, et al. Comparison of HASTE and segmented-HASTE sequences with a T2-weighted fast spin-echo sequence in the screening evaluation of the brain. AJR Am J Roentgenol. 1997;169(5):1401-1410.

\section{Disclosures}

Dr. Limbrick received support of a non-study-related clinical or research effort that he oversaw from Medtronic, Inc., and Microbot Medical, Inc. Dr. McKinstry received travel and meal expenses from Siemens Healthineers.

\section{Author Contributions}

Conception and design: Strahle, Smyth, Limbrick, Goyal, Shimony, McKinstry, Groves. Acquisition of data: Gewirtz, Skidmore. Analysis and interpretation of data: Gewirtz, Skidmore. Drafting the article: Gewirtz, Skidmore. Critically revising the article: Strahle, Gewirtz, Smyth, Goyal, Shimony, 
McKinstry. Reviewed submitted version of manuscript: all authors. Approved the final version of the manuscript on behalf of all authors: Strahle. Study supervision: Strahle.

\section{Supplemental Information}

Previous Presentations

This work was previously presented orally as an abstract at the American Association of Neurological Surgeons/Congress of Neurological Surgeons Joint Section on Pediatric Neurosurgery in December 2019.

\section{Correspondence}

Jennifer M. Strahle: Washington University in St. Louis, MO. strahlej@wustl.edu. 